\title{
Cosmological Perturbations in Antigravity
}

\author{
Marius Oltean* and Robert Brandenbergen ${ }^{\dagger}$ \\ Department of Physics, McGill University, Montreal, Quebec, H3A 2T8, Canada
}

(Dated: October 18, 2018)

\begin{abstract}
We compute the evolution of cosmological perturbations in a recently proposed Weyl-symmetric theory of two scalar fields with oppositely-signed conformal couplings to Einstein gravity. It is motivated from the minimal conformal extension of the Standard Model, such that one of these scalar fields is the Higgs while the other is a new particle, the dilaton, introduced to make the Higgs mass conformally symmetric. At the background level, the theory admits novel geodesically-complete cyclic cosmological solutions characterized by a brief period of repulsive gravity, or "antigravity," during each successive transition from a Big Crunch to a Big Bang. We show that despite the necessarily wrong-signed kinetic term of the dilaton in the full action, its cosmological solutions are stable at the perturbative level.
\end{abstract}

\section{INTRODUCTION}

The Big Bang singularity is arguably the most critical problem at the heart of modern cosmology. In the context of Einstein gravity minimally coupled to ordinary matter - the standard setting for the preponderance of current early Universe scenarios, such as inflation — initial singularities are unavoidable. To wit, their ubiquity has long been understood to be inextricably incurred by singularity theorems [1] which prove cosmological spacetimes therein to be, necessarily, geodesically-incomplete [2, 3].

Thus, in the present absence of a theory of quantum gravity, one potentially fruitful approach to constructing geodesically-complete cosmologies is the consideration of cosmological solutions to modified theories of (classical) gravity. In this paper, we will focus our attention on one particular such theory: namely, a scalar-tensor theory recently proposed and developed on the basis of Weyl symmetry [4 12] 24]. As its principal motivation, it has been argued to arise from the simplest Weyl-symmetric coupling of Einstein gravity to the minimal conformallyinvariant extension of the (classical) Standard Model [10. This leads (modulo the remaining particle content of the usual Standard Model) to an action for two scalars, the Higgs $s$ and a new field introduced to make its mass conformally symmetric, the dilaton $\phi$, each conformally coupled to Einstein gravity but with opposite signs. This allows for solutions to transit from a gravity phase to an antigravity phase and back 25.

While reducing to known physics in the expected limits (to ordinary Einstein gravity at low energies, and to the Standard Model in flat space), this theory entails new effects in regimes with strong gravity and large field fluctuations (when the amplitudes of $\phi$ and $s$ are comparable). Such conditions arise, quite naturally, in cosmology - specifically, in the vicinity of the Big Bang. Concordantly, a wealth of novel cosmological solutions have been obtained in this theory [8]; they are all geodesically-complete, and they all repeat cyclically (through Big Crunch-Big Bang transitions) [26].

The qualitative features [9] of these (homogeneous) solutions depend on whether or not anisotropies are included. For ease of discussion, we introduce the following definition for dividing the field space $\{(\phi, s)\}$ into two regions:

$$
\mathbb{G}_{ \pm}=\{(\phi, s):|\phi| \gtrless|s|\} .
$$

In other words, in $\mathbb{G}_{+}$, the amplitude of the Higgs is less than that of the dilaton; by construction of their respective conformal couplings, this corresponds to a positive Newton constant, and therefore gravity being attractive. Meanwhile, in $\mathbb{G}_{-}$, the amplitude of the Higgs is greater than that of the dilaton; this leads, accordingly, to a negative Newton constant, and therefore gravity being repulsive - also referred to as antigravity.

Without anisotropy, generic solutions (meaning, for varied choices of initial conditions and parameters of the model) cyclically pass through zero-size bounces: From a lengthy period in $\mathbb{G}_{+}$, they cross (at the Big Crunch) into a brief period in $\mathbb{G}_{-}$, and then exit it (at the following Big Bang) into another lengthy period in $\mathbb{G}_{+}$, and so on. A typical solution of this sort is the dotted green curve in Figure 1. (There is in fact also a special class of these solutions which can go through zero-size bounces - or even finite-size bounces, if they are spatial curvature-induced — without actually having to pass through $\mathbb{G}_{-}$.)

\footnotetext{
*Electronic address: moltean@physics.mcgill.ca
}

†Electronic address: rhb@hep.physics.mcgill.ca 
With anisotropy, an attractor mechanism is created (independent of initial conditions or choice of potential). All solutions are (cyclically) forced, after a lengthy period in $\mathbb{G}_{+}$, through the origin of field space (contracting to zero size at the Big Crunch), and then into a loop in $\mathbb{G}_{-}$, finally exiting it again through the origin (once more contracting to zero size) back into $\mathbb{G}_{+}$(leading to the usual expanding phase of attractive gravity following the Big Bang), and so on. A typical depiction of such a solution is the solid red curve in Figure 1 .

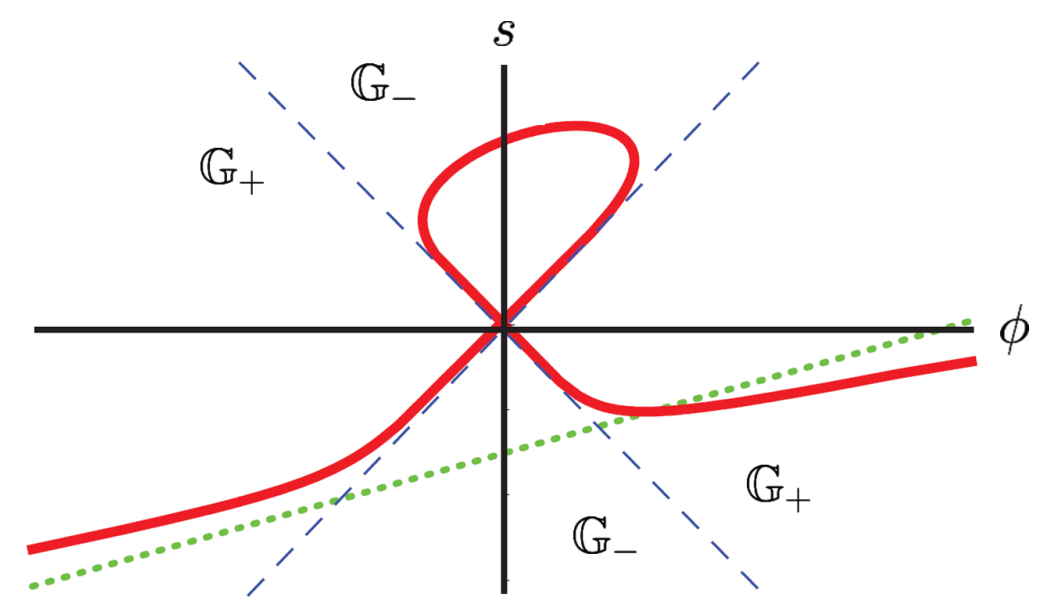

Figure 1: Taken from [8]. Generic background solutions without anisotropy (dotted green) and with anisotropy (solid red). The dashed blue lines bound the regions where gravity is attractive $\left(\mathbb{G}_{+}\right)$and repulsive $\left(\mathbb{G}_{-}\right)$.

Notwithstanding the merits of this theory insofar as it is able to resolve geodesic completeness in cosmology, the necessarily opposite signs of the conformal couplings of the two scalar fields constituting it immediately raises the question of ghosts, and the extent to which such may present any danger. In particular, although the Higgs kinetic term has the correct sign in the action (as it ought to), the kinetic term for the dilaton has the "wrong" sign, explicitly making it — by construction — a ghost. A priori, this need not be construed as problematic at least at the background level, since a unitary gauge choice (permitted by the Weyl symmetry) can be used to eliminate the ghost degree of freedom. It is not immediately obvious, however, if perturbations in this theory are themselves ghost-free. In other words, we cannot deduce right away whether the kinetic term of the second-order action will have the correct sign (nor the mass term, for that matter). If it does not, this would be problematic for the cosmological solutions of this theory [13. Thus, the principal aim of this paper will be to compute the evolution of its scalar perturbations with the motivating aim of establishing their stability (i.e. whether they are free of ghosts/tachyons). For simplicity, we will perform the analysis here in the absence of anisotropies, with potential set to zero and without any radiation. In addition, we do not consider the gravitational wave sector.

The remainder of this paper is structured as follows. In Section II we give the full action and equations of motion for this theory. Then, in Section III we develop our treatment of its cosmological perturbations. Following this, we analyze their stability in Section IV] and finally, in Section V] we offer concluding remarks.

\section{SETUP}

We work in the $(+,-,-,-)$ metric signature, and write all dimensionful quantities in Planck units.

As noted, the action for this theory comprises two scalar fields, the dilaton $\phi$ and the Higgs $s$, conformally coupled with opposite signs to Einstein gravity:

$$
S\left[\phi, s ; g_{\mu \nu}\right]=\int \mathrm{d}^{4} x \sqrt{-g}\left\{-\frac{\left(\phi^{2}-s^{2}\right)}{12} R-\frac{1}{2} g^{\mu \nu}\left(\nabla_{\mu} \phi \nabla_{\nu} \phi-\nabla_{\mu} s \nabla_{\nu} s\right)\right\} .
$$

A potential $V(\phi, s)$ for the two scalars may also be added to this, but for simplicity we will work with it set to zero.

The gravitational equation of motion is obtained by varying 2 with respect to the metric, which yields:

$$
\left(\phi^{2}-s^{2}\right) G_{\alpha \beta}+g_{\alpha \beta} g^{\mu \nu} \nabla_{\mu} \nabla_{\nu}\left(\phi^{2}-s^{2}\right)-\nabla_{\alpha} \nabla_{\beta}\left(\phi^{2}-s^{2}\right)=-6 T_{\alpha \beta},
$$

where on the right-hand side we have the usual stress-energy tensor of the two scalar fields (with appropriate opposite signs),

$$
T_{\alpha \beta}=-\frac{1}{2} g_{\alpha \beta} g^{\mu \nu}\left(\nabla_{\mu} \phi \nabla_{\nu} \phi-\nabla_{\mu} s \nabla_{\nu} s\right)+\left(\nabla_{\alpha} \phi \nabla_{\beta} \phi-\nabla_{\alpha} s \nabla_{\beta} s\right) .
$$


Meanwhile, varying (2) with respect to either of the two scalars produces the same matter equation of motion,

$$
\phi g^{\mu \nu} \nabla_{\mu} \nabla_{\nu} s-s g^{\mu \nu} \nabla_{\mu} \nabla_{\nu} \phi=0
$$

The fact that there is not a separate equation of motion for each scalar field happens to be a consequence of the Weyl symmetry along with the fact that we have chosen a vanishing potential [4].

\section{COSMOLOGICAL PERTURBATIONS}

We work in the conformal Newtonian gauge, and assume that there is no anistropic stress at linear order in the matter fluctuations. This allows us to employ the perturbed Friedmann-Robertson-Walker metric

$$
g_{\mu \nu}=a^{2}(\tau)\left[\eta_{\mu \nu}+2 \varepsilon \Phi(\tau, \mathbf{x}) \delta_{\mu \nu}\right]
$$

where $\eta_{\mu \nu}=\operatorname{diag}(1,-1,-1,-1)$ is the Minkowski metric, $\Phi$ is the scalar metric perturbation, and we use $\varepsilon$ as our perturbation parameter (so that $\mathcal{O}\left(\varepsilon^{n}\right)$ is the $n$-th perturbative order). Moreover, we consider perturbed expressions for the two matter fields,

$$
\left\{\begin{array}{l}
\phi(\tau, \mathbf{x})=\phi_{0}(\tau)+\varepsilon \Pi(\tau, \mathbf{x}) \\
s(\tau, \mathbf{x})=s_{0}(\tau)+\varepsilon \Theta(\tau, \mathbf{x})
\end{array}\right.
$$

where $\phi_{0}$ and $s_{0}$ are the background (i.e. homogeneous) solutions for the dilaton and Higgs fields, while $\Pi$ and $\Theta$ are their perturbations, respectively.

Furthermore, it will be useful for later convenience to define the following background variables:

$$
\left\{\begin{array}{l}
z=\phi_{0}^{2}-s_{0}^{2} \\
\tilde{z}=\phi_{0}^{\prime 2}-s_{0}^{\prime 2}
\end{array}\right.
$$

as well as perturbation variables:

$$
\left\{\begin{array} { l } 
{ \alpha = \phi _ { 0 } \Pi - s _ { 0 } \Theta , } \\
{ \tilde { \alpha } = \phi _ { 0 } ^ { \prime } \Pi ^ { \prime } - s _ { 0 } ^ { \prime } \Theta ^ { \prime } , }
\end{array} \text { and } \quad \left\{\begin{array}{l}
\Upsilon=z \Phi-\alpha, \\
\tilde{\Upsilon}=\tilde{z} \Phi-\tilde{\alpha}
\end{array}\right.\right.
$$

We can now proceed to studying the evolution of these perturbations. We approach this both from the point of view of the equations of motion, and of the action. As a consistency check, we show in Appendix A that all of our results detailed in the following subsections correctly reduce to those of the standard theory of cosmological perturbations in Einstein gravity in the appropriate limit.

\section{A. Perturbed Equations of Motion}

We derive the perturbed gravitational and matter equations of motion by directly inserting (6) and (7) into (3) and (5). We state the results in Table I making use of the variables (8) and (9) defined above. In the case of Einstein gravity, the off-diagonal space-space equations are trivial in the absence of anisotropic stress. This is not the case here. The off-diagonal space-space equation of motion reads

$$
\phi_{0} \partial_{i} \partial_{j} \Pi-s_{0} \partial_{i} \partial_{j} \Theta=0 \text {. }
$$

We shall assume that this constraint equation is satisfied. This assumption is only possible to make because we have two matter fields. In the case of a single non-canonically coupled matter field this equation cannot be satisfied and leads to a paradox [14.

While direct computation of these equations offers one possible approach to studying the evolution of perturbations in this theory, they are not particularly illuminating especially when it comes to the question of how we might reduce the number of propagating degrees of freedom at the perturbative level from the current three (the metric perturbation $\Phi$, the dilaton perturbation $\Pi$ and the Higgs perturbation $\Theta$ ). More helpful in this regard will be to compute the second-order action. 


\begin{tabular}{|c|c|c|}
\hline EOM & $\mathcal{O}(1)$ & $\mathcal{O}(\varepsilon)$ \\
\hline (3) time-time & $0=z \mathcal{H}^{2}+z^{\prime} \mathcal{H}+\tilde{z}$ & $0=\nabla^{2} \Upsilon-3 \mathcal{H}\left(\Upsilon^{\prime}+\Upsilon \mathcal{H}\right)-3 \tilde{\Upsilon}-\frac{3}{2} z^{\prime} \Phi^{\prime}$ \\
\hline (3) space-space & $\begin{aligned} 0= & z\left(2 \mathcal{H}^{\prime}+\mathcal{H}^{2}\right) \\
& +z^{\prime} \mathcal{H}+z^{\prime \prime}-3 \tilde{z}\end{aligned}$ & $\begin{aligned} 0= & \Upsilon^{\prime \prime}+\mathcal{H}\left(\Upsilon^{\prime}+2 z \Phi^{\prime}\right)+\frac{1}{2} z^{\prime} \Phi^{\prime} \\
& +\left(2 \mathcal{H}^{\prime}+\mathcal{H}^{2}\right) \Upsilon-3 \tilde{\Upsilon}+\frac{2}{3} \nabla^{2} \alpha\end{aligned}$ \\
\hline (3) time-space & Trivial. & $\begin{aligned} 0=\partial_{i} & {\left[z\left(\mathcal{H} \Phi+\Phi^{\prime}\right)+\frac{1}{2} z^{\prime} \Phi+\mathcal{H} \alpha-\alpha^{\prime}\right.} \\
& \left.+3\left(\phi_{0}^{\prime} \Pi-s_{0}^{\prime} \Theta\right)\right]\end{aligned}$ \\
\hline 5 & $\begin{aligned} 0= & \phi_{0}\left(s_{0}^{\prime \prime}+2 \mathcal{H} s_{0}^{\prime}\right) \\
& -s_{0}\left(\phi_{0}^{\prime \prime}+2 \mathcal{H} \phi_{0}^{\prime}\right)\end{aligned}$ & $\begin{aligned} 0= & \phi_{0}\left(\Theta^{\prime \prime}-\nabla^{2} \Theta+2 \mathcal{H} \Theta^{\prime}-4 s_{0}^{\prime} \Phi^{\prime}\right) \\
& -s_{0}\left(\Pi^{\prime \prime}-\nabla^{2} \Pi+2 \mathcal{H} \Pi^{\prime}-4 \phi_{0}^{\prime} \Phi^{\prime}\right) \\
& -\left(\phi_{0}^{\prime \prime}+2 \mathcal{H} \phi_{0}^{\prime}\right) \Theta+\left(s_{0}^{\prime \prime}+2 \mathcal{H} s_{0}^{\prime}\right) \Pi\end{aligned}$ \\
\hline
\end{tabular}

Table I: Perturbed equations of motion.

\section{B. Perturbed Action}

Inserting (6) and (7) into (2) and simplifying the result as much as possible via integration by parts and substitution of background equations (i.e. the $\mathcal{O}(1)$ equations from Table I), we obtain the following secondorder action:

$$
\begin{aligned}
S^{(2)}=\varepsilon^{2} \int \mathrm{d}^{4} x \frac{a^{2}}{2}\{ & -z\left[\Phi^{\prime 2}+\frac{1}{3}(\nabla \Phi)^{2}\right]-\left[\Pi^{\prime 2}-\Theta^{\prime 2}\right]+\left[(\nabla \Pi)^{2}-(\nabla \Theta)^{2}\right]+\left(\mathcal{H}^{2}+\mathcal{H}^{\prime}\right)\left[\Pi^{2}-\Theta^{2}\right] \\
& \left.-\frac{2}{3}(\nabla \alpha \cdot \nabla \Phi)+2 \Phi^{\prime}\left(\alpha^{\prime}+2 \mathcal{H} \alpha\right)+8 \Phi\left[\mathcal{H}\left(\alpha^{\prime}+\mathcal{H} \alpha\right)+\tilde{\alpha}\right]\right\}
\end{aligned}
$$

As expected, we have three kinetic terms corresponding to each of the three perturbation variables. We can now try to further simplify this with the help of the time-space gravitational equation.

\section{From Two to One Matter Perturbation}

Consider the (3) time-space $\mathcal{O}(\varepsilon)$ equation from Table I] which we rewrite more suggestively as:

$$
\phi_{0} \Pi^{\prime}-s_{0} \Theta^{\prime}=\frac{1}{3}\left[z\left(\mathcal{H} \Phi+\Phi^{\prime}\right)+\frac{1}{2} z^{\prime} \Phi+\mathcal{H} \alpha+2 \alpha^{\prime}\right] .
$$

This indicates that one out of the three propagating degrees of freedom appearing in (11) can in fact be expressed in terms of the other two; in other words, it should be possible to get the perturbed action in terms of only two perturbation variables. To do this, we proceed as follows: We write $\Pi=\left(\alpha+s_{0} \Theta\right) / \phi_{0}$ (by the definition $(9)$ of $\alpha)$, take its time derivative, and insert the resulting expression for $\Pi^{\prime}$ into $(12)$. The $\Theta^{\prime}$ terms cancel, and we get an expression for $\Theta$,

$$
\Theta=\left[\frac{z \mathcal{H}+\frac{1}{2} z^{\prime}}{3 \phi_{0}\left(s_{0} / \phi_{0}\right)^{\prime}}\right] \Phi+\left[\frac{z}{3 \phi_{0}\left(s_{0} / \phi_{0}\right)^{\prime}}\right] \Phi^{\prime}-\left[\frac{\alpha}{3 \phi_{0}\left(s_{0} / \phi_{0}\right)^{\prime}}\right]^{\prime}+\left[3\left(\frac{\phi_{0}^{\prime}}{\phi_{0}}+\mathcal{H}\right)+\frac{\phi_{0}^{\prime}}{\phi_{0}}\right] \frac{\alpha}{3 \phi_{0}\left(s_{0} / \phi_{0}\right)^{\prime}}
$$

simply in terms of $\Phi, \alpha$, and their derivatives. Then, $\Pi$ can in turn be written in terms of the same by putting this expression for $\Theta$ into $\Pi=\left(\alpha+s_{0} \Theta\right) / \phi_{0}$.

To make these expressions for the matter perturbations cleaner, as well as for future convenience, we define a list of additional background variables in Table II

Now, (13) readily suggests that we work not with the variable $\alpha=\phi_{0} \Pi-s_{0} \Theta$, but instead with

$$
\psi=\frac{\alpha}{3 \phi_{0} u^{\prime}}=\frac{\phi_{0} \Pi-s_{0} \Theta}{3 \phi_{0}\left(s_{0} / \phi_{0}\right)^{\prime}} .
$$




\begin{tabular}{|l||l|}
\hline$u=\frac{s_{0}}{\phi_{0}}$ & $v=\frac{\phi_{0}^{\prime}}{\phi_{0}}$ \\
\hline$\tilde{u}=1-u^{2}=\frac{z}{\phi_{0}^{2}}$ & $\tilde{v}=v+\mathcal{H}$ \\
\hline $\bar{u}=\frac{\phi_{0} \tilde{u}}{6 u^{\prime}}$ & $\bar{v}=4 \tilde{v} v-2 \tilde{v}^{\prime}+\mathcal{H}^{2}+\mathcal{H}^{\prime}$ \\
\hline$\hat{u}=v \tilde{u}^{\prime}-\left(\mathcal{H}^{2}+\mathcal{H}^{\prime}\right) \tilde{u}$ & $\hat{v}=6\left(\tilde{v} \tilde{u}^{\prime}-u^{\prime 2}\right)$ \\
\hline
\end{tabular}

Table II: Useful background variables.

Combining all of the above, we can express both (dilaton and Higgs) matter perturbations simply in terms of the metric perturbation $\Phi$, the new variable $\psi$, and their derivatives (as well as background variables):

$$
\left\{\begin{array}{l}
\Pi=u \Theta+3 u^{\prime} \psi, \\
\Theta=2 \bar{u} \Phi^{\prime}+\left(\bar{u}^{\prime}-v \bar{u}\right) \Phi-\psi^{\prime}+(3 \tilde{v}+v) \psi .
\end{array}\right.
$$

Henceforth, we can regard $\psi$ as being the (single) variable describing matter perturbations.

\section{Perturbed Action in Two Variables}

The next step is to insert 15 into the second order action (11) and thereby write the latter purely in terms of $\Phi$ and $\psi$.

The first step in the simplification is to get rid of the $\tilde{\alpha}$ term. To do this, we insert its definition (9) in terms of the original perturbation variables, $\tilde{\alpha}=\phi_{0}^{\prime} \Pi^{\prime}-s_{0}^{\prime} \Theta^{\prime}$, integrate it by parts, and then use 12 to simplify:

$$
\begin{aligned}
S^{(2)}=\varepsilon^{2} \int \mathrm{d}^{4} x \frac{a^{2}}{2}\{ & -\Pi^{\prime 2}+\Theta^{\prime 2}+\left[(\nabla \Pi)^{2}-(\nabla \Theta)^{2}\right]+\left[\left(\mathcal{H}^{2}+\mathcal{H}^{\prime}\right)\left(\Pi^{2}-\Theta^{2}\right)\right]+z\left[\frac{5}{3} \Phi^{\prime 2}-\frac{1}{3}(\nabla \Phi)^{2}\right] \\
& \left.-\frac{2}{3}(\nabla \alpha \cdot \nabla \Phi)-\frac{2}{3} \Phi^{\prime} \alpha^{\prime}-\frac{4}{3} \mathcal{H} \Phi^{\prime} \alpha-8\left[v^{\prime}+(v+2 \mathcal{H}) v+\mathcal{H}^{2}+\mathcal{H}^{\prime}\right] \Phi \alpha\right\} .
\end{aligned}
$$

To make progress, we set at this point all spatial gradients to zero. Then, writing $\alpha$ in terms of the more convenient variable $\psi$ defined by $[14$, the above becomes:

$$
\begin{aligned}
S^{(2)}=\varepsilon^{2} \int \mathrm{d}^{4} x \frac{a^{2}}{2}\{ & -\Pi^{\prime 2}+\Theta^{\prime 2}+\left[\mathcal{H}^{2}+\mathcal{H}^{\prime}\right]\left(\Pi^{2}-\Theta^{2}\right)+\frac{5}{3} z \Phi^{\prime 2} \\
& \left.-2 \phi_{0} u^{\prime} \Phi^{\prime}\left(\psi^{\prime}-v \psi\right)-24 \phi_{0} u^{\prime}\left[\tilde{v}^{\prime}+\tilde{v}^{2}\right] \Phi \psi\right\} .
\end{aligned}
$$

Now, we can write $\Pi$ in terms of $\Theta$ using the first expression in 15:

$$
\begin{gathered}
S^{(2)}=\varepsilon^{2} \int \mathrm{d}^{4} x \frac{a^{2}}{2}\left\{\tilde{u} \Theta^{\prime 2}+\hat{u} \Theta^{2}-3 \bar{v} \tilde{u}^{\prime} \psi \Theta+\hat{v} \psi^{\prime} \Theta+3 \tilde{u}^{\prime} \psi^{\prime} \Theta^{\prime}-9 u^{\prime 2} \psi^{\prime 2}+9 \bar{v} u^{\prime 2} \psi^{2}\right. \\
\left.+\frac{5}{3} z \Phi^{\prime 2}-2 \phi_{0} u^{\prime} \Phi^{\prime}\left(\psi^{\prime}-v \psi\right)-24 \phi_{0} u^{\prime}\left[\tilde{v}^{\prime}+\tilde{v}^{2}\right] \Phi \psi\right\} .
\end{gathered}
$$

Finally, we could substitute into this our second expression from 115 for $\Theta$ in terms of $\Phi, \psi$ and their derivatives, in order to get $S^{(2)}$ entirely in terms of the latter two variables. However, there is an issue with this: Since $\Theta$ contains $\Phi^{\prime}$ and $\psi^{\prime}$ terms, and since $S^{(2)}$ contains $\Theta^{\prime 2}$ terms, this will lead to $\Phi^{\prime \prime 2}$ and $\psi^{\prime \prime 2}$ terms in the action. Instead, we would like to have just standard kinetic-type (i.e. single time derivative squared) terms. Accordingly, this difficulty immediately suggests a possible resolution.

\section{Field Redefinitions and Diagonalization}

If we were to define a new field as a linear combination of $\Phi$ and $\Phi^{\prime}$, then the $\Phi^{\prime \prime 2}$ term appearing in $S^{(2)}$ might simply be construed as a contribution to its (standard) kinetic-type term - and similarly in the case of $\psi$. Alternately, we can take $\Phi^{\prime}$ to be a linear combination of the new field and $\Phi$ - and again, similarly for $\psi$. 
Following this logic, let $A, B, C$, and $D$ be (as of yet) undetermined functions of time, and define two new fields $\tilde{\Phi}$ and $\tilde{\psi}$ according to $\Phi^{\prime}=A \tilde{\Phi}+B \Phi$ and $\psi^{\prime}=C \tilde{\psi}+D \psi$, or:

$$
\left\{\begin{array}{l}
\tilde{\Phi}=\frac{1}{A} \Phi^{\prime}-\frac{B}{A} \Phi \\
\tilde{\psi}=\frac{1}{C} \psi^{\prime}-\frac{D}{C} \psi
\end{array}\right.
$$

Moreover, it will be useful to define

$$
\tilde{A}=2 \bar{u} A, \quad \tilde{B}=\bar{u}^{\prime}+(2 B-v) \bar{u}, \quad \tilde{C}=-C, \quad \tilde{D}=3 \tilde{v}+v-D,
$$

so that

$$
\Theta=\tilde{A} \tilde{\Phi}+\tilde{B} \Phi+\tilde{C} \tilde{\psi}+\tilde{D} \psi
$$

Now, if we insert this version of $\Theta$ into 18 and rewrite, as much as possible, the remaining terms that contain $\Phi^{\prime}$ and $\psi^{\prime}$ in terms of $\tilde{\Phi}$ and $\tilde{\psi}$, we can simplify $S^{(2)}$ into a form that contains only the $\tilde{\Phi}$ and $\tilde{\psi}$ perturbation variables plus four terms that do not: a $\Phi^{2}$ term, a $\psi^{2}$ term, a $\Phi \psi$ term and a $\Phi^{\prime} \psi$ term. The coefficients of these four terms, constructed out of the four functions $A, B, C$, and $D$ and their derivatives, we can then choose to set to zero. In other words, provided $A, B, C$, and $D$ are any solutions satisfying the set of four ODEs resulting from the demand that these four coefficients vanish, then all terms involving $\Phi$ and $\psi$ can be eliminated - meaning that we can write $S^{(2)}$ purely in terms of the new fields $\tilde{\Phi}$ and $\tilde{\psi}$. The result is:

$$
S^{(2)}=\varepsilon^{2} \int \mathrm{d}^{4} x \frac{a^{2}}{2}\left\{\tilde{u} \tilde{A}^{2} \tilde{\Phi}^{\prime 2}+\tilde{u} \tilde{C}^{2} \tilde{\psi}^{\prime 2}+2 \tilde{u} \tilde{A} \tilde{C} \tilde{\Phi}^{\prime} \tilde{\psi}^{\prime}+p \tilde{\Phi}^{\prime} \tilde{\psi}+2 q \tilde{\Phi} \tilde{\psi}+m_{1} \tilde{\Phi}^{2}+m_{2} \tilde{\psi}^{2}\right\}
$$

where the coefficients $p, q, m_{1}$ and $m_{2}$ are given in terms of $A, B, C$, and $D$ in Appendix B. Moreover, the full set of ODEs that the latter need to satisfy are also given there. They are highly nontrivial, and we make no attempt to solve them - but they do, at the very least, imply that solutions for $A, B, C$, and $D$ such that the second order action acquires the desired canonical form 22 exist.

The point can here be raised that it may appear as though we've eliminated degrees of freedom in going from the second order action in terms of $\Phi$ and $\psi$ to its canonical version in terms of $\tilde{\Phi}$ and $\tilde{\psi}$ - specifically, those contained in the squared double derivative terms. We can, rather, think of these degrees of freedom as having been absorbed into the time dependent functions $A, B, C$, and $D$. One way to look at this is as follows: If we had kept $S^{(2)}$ in terms of $\Phi$ and $\psi$, we would end up with equations of motion in the form of third-order ODEs, whose solutions could in principle be obtained after the background is solved for. Instead, we redefined everything in terms of the new fields $\tilde{\Phi}$ and $\tilde{\psi}$, which (due to the now canonical form of $S^{(2)}$ ) obey second-order ODEs. Yet, this can be achieved only on the condition that the functions $A, B, C$, and $D$ permitting this redefinition themselves obey a set of ODEs (that given in Appendix B which, indeed, turn out to be third-order differential equations - the solutions for which, again, depend on the background.

Next, we diagonalize the kinetic term. We present the details of the procedure in Appendix C and here simply state the result. Performing a field redefinition

$$
\left\{\begin{array}{l}
\zeta=\tilde{A} \tilde{\Phi}+\tilde{C} \tilde{\psi} \\
\xi=-\tilde{C} \tilde{\Phi}+\tilde{A} \tilde{\psi}
\end{array}\right.
$$

the second order action 22 becomes:

$$
S^{(2)}=\varepsilon^{2} \int \mathrm{d}^{4} x \frac{a^{2}}{2}\left\{\tilde{u} \zeta^{\prime 2}+c_{1} \zeta^{2}+c_{2} \xi^{2}+c_{3} \zeta \xi+c_{4} \zeta^{\prime} \xi\right\}
$$

with $c_{1}, c_{2}, c_{3}$ and $c_{4}$ given explicitly in Appendix C. Thus, we see that there is in fact only one propagating degree of freedom, namely the field $\zeta$.

We can simplify this further still, by solving for the non-propagating field $\xi$ and substituting it back into the action. Its equation of motion is found by varying (24) with respect to $\xi$ and simply yields:

$$
\xi=-\frac{1}{2 c_{2}}\left(c_{3} \zeta+c_{4} \zeta^{\prime}\right)
$$

Inserting this back into 24), expanding, and integrating by parts, we obtain a remarkably simple (modulo coefficient determination) second order action:

$$
S^{(2)}=\varepsilon^{2} \int \mathrm{d}^{4} x \frac{a^{2}}{2}\left\{C_{1} \zeta^{\prime 2}+C_{2} \zeta^{2}\right\}
$$


where

$$
\begin{aligned}
& C_{1}=\tilde{u}-\frac{c_{4}^{2}}{4 c_{2}} \\
& C_{2}=c_{1}+\frac{c_{3}}{4 c_{2}}\left[2 \mathcal{H} c_{4}-c_{3}\right]+\left(\frac{c_{3} c_{4}}{4 c_{2}}\right)^{\prime} .
\end{aligned}
$$

Unfortunately, as an exact analytical computation of $C_{1}$ and $C_{2}$ seems to be (at least with our current approach) an immensely nontrivial problem, (26) as such can only give us the form of the equation of motion for perturbations in this theory, but not an indication as to whether or not solutions thereof are in general stable. In other words, we cannot immediately conclude from 26 if $\zeta$ is ghost-like nor tachyonic. Instead, we shall have to resort to approximations, which we turn to in the next section.

\section{STABILITY ANALYSIS}

The first step in determining whether cosmological solutions of this theory are perturbatively stable is to ask where we would expect things to go wrong. We can infer right away that we need not be concerned when they pass through the attractive gravity region $\mathbb{G}_{+}$of field space. The reason is simply that a gauge choice (in the form of a judicious reparametrization of the matter fields, as described in Appendix A can here be made to reduce the theory to ordinary (attractive) Einstein gravity. Therefore, the regime where we might worry about the possible appearance of perturbative ghosts (or tachyons) is the repulsive gravity region $\mathbb{G}_{-}$- this being, indeed, where all the novel effects of the theory come in.

In particular, we will focus our attention on the evolution of perturbations around the area of $\mathbb{G}_{-}$where the background dilaton becomes very small, while the background Higgs does not. In other words, we will consider the limit in which $\phi_{0} \rightarrow 0$, but $s_{0}$ as well as their first derivatives remain finite. This corresponds to the regime in field space where the Newton constant attains its most negative value, and so for this reason we refer to it as the deep antigravity limit.

We will base our overall determination of the stability of perturbations in this theory upon their behaviour in this limit, for two reasons. First, it is a limit which all background solutions passing through $\mathbb{G}_{-}$necessarily exhibit at some point (irrespective of whether they contain anisotropies, which we have not even included in our analysis here) and which, as we shall see in the next subsection, can be taken via a more or less straightforward procedure. Second, though a verdict on stability/instability in the deep antigravity regime need not translate to the same throughout all of $\mathbb{G}_{-}$, we can offer an argument for why it is nonetheless sufficiently indicative for allowing us to draw a conclusion on the entire theory. In the case that a ghost/tachyon is found here, we would immediately deduce that the theory is problematic (or, at best, contingent upon the instability having a very short duration). In the case that it is not found, we are justified in asserting that the theory is safe. The reason for this is as follows: Even if a perturbative instability actually does develop at any other point during the passage through $\mathbb{G}_{-}$(e.g. at the boundary between $\mathbb{G}_{+}$and $\mathbb{G}_{-}$), it would have to be so short-lived as to be quickly back under control by the (ghost-free) deep antigravity regime roughly corresponding to the half-way point of the (already, generically brief) repulsive gravity phase. In other words, even if the kinetic term (or mass term) reversed signs anywhere else during $\mathbb{G}_{-}$but went back to the correct sign about half-way through it, this potential momentary instability may not be regarded as so catastrophic as to jeopardize the entire theory.

In summary: To establish the stability of perturbations in this theory, it will suffice to take the deep antigravity limit of the second-order action, i.e. $\phi_{0} \rightarrow 0$ while keeping all other background quantities finite.

The second step in addressing the issue of stability is to ask which of the perturbation variables we should watch out for. Seeing as one of the two matter fields (namely the dilaton) is what appears as a (potentially problematic) ghost in the full action, it is the matter perturbation that is the sole concern here (while the metric perturbation's stability should not have to worry us). In other words, in lieu of determining whether the single perturbation variable $\zeta$ is stable, it will suffice to try to find whether $\psi$ is stable. Of course, taking the deep antigravity limit in the second-order action 26 for the former is made difficult by the fact that we have no analytic expression - nor, indeed, even a good ansatz - for the functions $A, B, C$ and $D$ upon which the coefficients appearing therein ultimately depend. But we can take this limit in the case of the latter - in particular, by looking at the last version of the second-order action we obtained just before these four functions made their appearance. 


\section{A. Deep Antigravity Approximation}

Consider $S^{(2)}$ in the form 18 just prior to making the field redefinitions to remove the squared double derivative terms. We rewrite it here for convenience:

$$
\begin{gathered}
S^{(2)}=\varepsilon^{2} \int \mathrm{d}^{4} x \frac{a^{2}}{2}\left\{\tilde{u} \Theta^{\prime 2}+\hat{u} \Theta^{2}-3 \bar{v} \tilde{u}^{\prime} \psi \Theta+\hat{v} \psi^{\prime} \Theta+3 \tilde{u}^{\prime} \psi^{\prime} \Theta^{\prime}-9 u^{\prime 2} \psi^{\prime 2}+9 \bar{v} u^{\prime 2} \psi^{2}\right. \\
\left.+\frac{5}{3} z \Phi^{\prime 2}-2 \phi_{0} u^{\prime} \Phi^{\prime}\left(\psi^{\prime}-v \psi\right)-24 \phi_{0} u^{\prime}\left[\tilde{v}^{\prime}+\tilde{v}^{2}\right] \Phi \psi\right\},
\end{gathered}
$$

where $\Theta$ should be everywhere understood according to 15 as written in terms of the metric perturbation $\Phi$ and matter perturbation $\psi$, namely:

$$
\Theta=2 \bar{u} \Phi^{\prime}+\left(\bar{u}^{\prime}-v \bar{u}\right) \Phi-\psi^{\prime}+(3 \tilde{v}+v) \psi .
$$

The advantage of taking the $\phi_{0} \rightarrow 0$ limit of 29 (as opposed to the second-order action $(26)$ for $\zeta$ ) is that all of the coefficients are known exactly. The strategy then is to insert their full expressions and collect the result in powers of $\phi_{0}$. The lowest order (or highest inverse order) terms will be the dominant ones, and therefore the ones of interest.

Now, extracting their explicit forms from Table II, we note that all of the coefficient functions appearing in (29) with (30) substituted are in fact exact (finite) series in powers of $\phi_{0}$, with the sole exception of the function $u$. Taylor expanding the latter in $\phi_{0}$, inserting the exact expressions for the rest of the coefficients, collecting the integrand in powers of $\phi_{0}$, integrating by parts and simplifying as much as possible, we get:

$$
S^{(2)}=\varepsilon^{2} \int \mathrm{d}^{4} x \frac{a^{2}}{2}\left\{\left[-\frac{24 \phi_{0}^{\prime 4} s_{0}^{2}}{\phi_{0}^{6}}+\cdots\right] \psi^{2}+\frac{3 \phi_{0}^{\prime 2} s_{0}^{2}}{\phi_{0}^{4}} \psi^{\prime 2}+\cdots\right\},
$$

where $\cdots$ here refers to higher order terms in $\phi_{0}$. These include all contributions from the $\Phi$ perturbation as well as the squared double derivative terms, which accordingly do not concern us.

We thus see that the significant contributions to the second order action in the deep antigravity limit are simply a kinetic term and a mass term for the matter perturbation variable $\psi$. If we write $(31)$ more suggestively (dropping the higher order terms) as

$$
S^{(2)}=\varepsilon^{2} \int \mathrm{d}^{4} x\left(\frac{\sqrt{3} a \phi_{0}^{\prime} s_{0}}{\sqrt{2} \phi_{0}^{2}}\right)^{2}\left\{\psi^{\prime 2}-\left(2 \sqrt{2} \frac{\phi_{0}^{\prime}}{\phi_{0}}\right)^{2} \psi^{2}\right\},
$$

we see that $\psi$ has the correct (positive) sign for its kinetic term, and also the correct (negative) sign for its mass term. This means, respectively, that it is neither a ghost nor a tachyon. Hence, based on our entire discussion in this section up to now, we conclude that cosmological solutions of this theory are perturbatively stable, and therefore viable.

\section{B. Equation of Motion and Solution}

Finally, we derive the equation of motion for the perturbation in this limit, and obtain its solution. Varying the approximated second-order action $(31)$ with respect to $\psi$ yields:

$$
0=\psi^{\prime \prime}+\left(-4 \frac{\phi_{0}^{\prime}}{\phi_{0}}+\cdots\right) \psi^{\prime}+\left(2 \sqrt{2} \frac{\phi_{0}^{\prime}}{\phi_{0}}+\cdots\right)^{2} \psi
$$

To solve this, we can insert the background solution for $\phi_{0}$ obtained in [8]:

$$
\phi_{0}(\eta)=\mathscr{A} \frac{\operatorname{sn}(\eta, m)}{\operatorname{dn}(\eta, m)},
$$

where $\eta=\left(\tau+\tau_{0}\right) / T$ is a shifted and rescaled time coordinate, $\mathscr{A}$ is a constant, and sn and dn are Jacobi elliptic functions, with the parameter $m=1 / 2$.

To get a better intuition for (34), we can look at its Taylor series in $\eta$ :

$$
\phi_{0}=\mathscr{A}\left[\eta-\frac{1}{12} \eta^{3}-\frac{1}{60} \eta^{5}+\mathcal{O}\left(\eta^{7}\right)\right] .
$$


So we see that the $\phi_{0} \rightarrow 0$ limit corresponds to $\eta \rightarrow 0$. Thus, we can use the approximation $\phi_{0} \approx \mathscr{A} \eta$ in this regime.

At this point we can take two possible approaches towards solving (33). One is to directly insert (34) into it, as is. The resulting ODE actually turns out to have an exact solution. However, its form is not particularly illuminating; it is given in terms of Jacobi elliptic functions and the Heun general function. For the sake of completeness, we write it down in full in Appendix D

A second, and perhaps more instructive approach to solving $(33)$ is to make some further approximations first. As suggested, we can try to use $\phi_{0} \approx \mathscr{A} \eta$, which entails $\left(\mathrm{d} \phi_{0} / \mathrm{d} \eta\right) / \phi_{0} \approx 1 / \eta$. With this, our ODE for $\psi$, written in terms of the variable $\eta$, becomes:

$$
0=\frac{\mathrm{d}^{2} \psi}{\mathrm{d} \eta^{2}}-\frac{4}{\eta} \frac{\mathrm{d} \psi}{\mathrm{d} \eta}+\frac{8}{\eta^{2}} \psi
$$

which is easily solvable. Its two independent solutions are:

$$
\left\{\begin{array}{l}
\psi_{1}=|\eta|^{5 / 2} \sin \left(\frac{\sqrt{7}}{2} \ln |\eta|\right), \\
\psi_{2}=|\eta|^{5 / 2} \cos \left(\frac{\sqrt{7}}{2} \ln |\eta|\right) .
\end{array}\right.
$$

We plot each of these in Figure 2

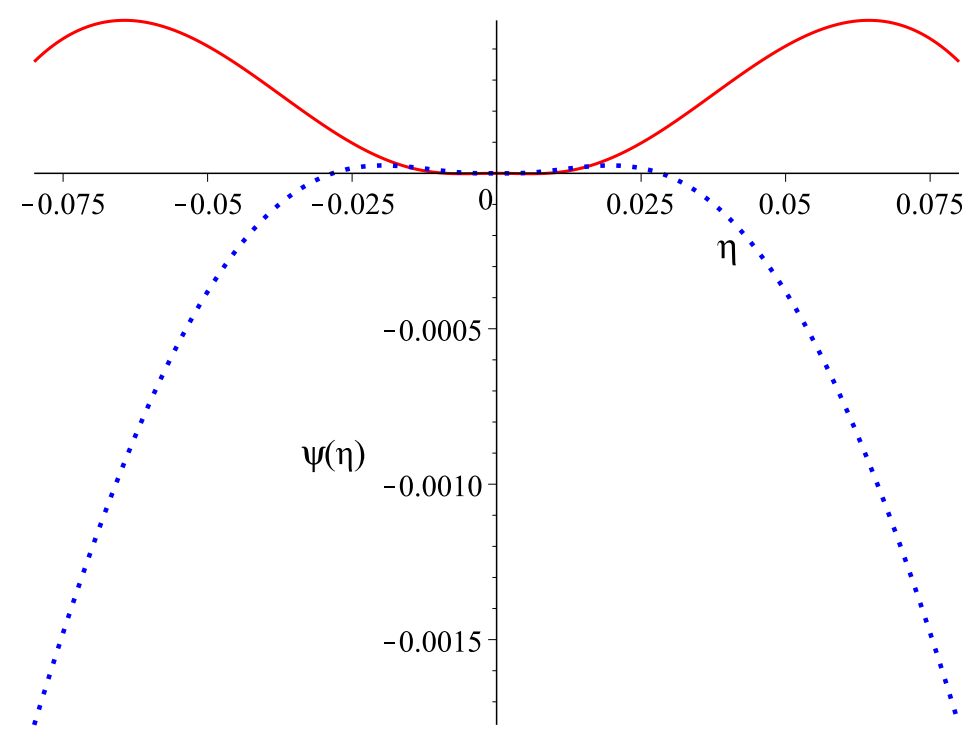

Figure 2: The two independent solutions for the matter perturbation in the deep antigravity regime: $\psi_{1}$ (solid red) and $\psi_{2}$ (dotted blue).

The general solution is then a superposition of the two:

$$
\psi=|\eta|^{5 / 2}\left[\mathscr{C}_{1} \sin \left(\frac{\sqrt{7}}{2} \ln |\eta|\right)+\mathscr{C}_{2} \cos \left(\frac{\sqrt{7}}{2} \ln |\eta|\right)\right]
$$


where $\mathscr{C}_{1}$ and $\mathscr{C}_{2}$ are constants.

We remark that, for all solutions, $\psi \rightarrow 0$ as $\eta \rightarrow 0$. That is to say, perturbations become suppressed in the deep antigravity regime. This behaviour is markedly contrary to what happens in ordinary (Einstein) gravity. There, it is precisely the fact that gravity is attractive which leads to the growth of inhomogeneities, and consequently, structure formation: In brief, overdensities increase because their surrounding matter is further attracted into them [15, 16]. When gravity is strongly repulsive, on the other hand, what may start out as an overdensity will soon be repelled apart. What is more, we see here that when gravity is as repulsive as it can possibly be, matter becomes evenly repelled to the point that any prior fluctuation will completely vanish, leaving us with an entirely homogeneous Universe. This suggests that this theory may be able to supply its own resolution to the problem of why, indeed, we observe our own Universe to be so nearly homogeneous today.

\section{CONCLUSIONS}

\section{A. Summary of Results}

In this paper, we have considered the Weyl-symmetric theory (2) of two scalar fields, the dilaton $\phi$ and the Higgs $s$, conformally coupled with opposite signs to Einstein gravity, and we have shown that its isotropic cosmological solutions admit scalar perturbations whose second-order action $(26)$ can in general be written in a canonical form,

$$
S^{(2)}=\varepsilon^{2} \int \mathrm{d}^{4} x \frac{a^{2}}{2}\left\{C_{1} \zeta^{\prime 2}+C_{2} \zeta^{2}\right\}
$$

in terms of a single propagating perturbation variable $\zeta$, which is a linear combination of the metric and matter perturbations, and their first derivatives.

Moreover, we have studied this theory in the regime where the dilaton approaches zero but the Higgs remains finite (corresponding to the epoch of strongly repulsive gravity roughly mid-way between a Big Crunch and a Big Bang in its cosmological solutions), and found that the second-order action (31) there, which can be approximated purely in terms of the single matter perturbation $\psi$,

$$
S^{(2)}=\varepsilon^{2} \int \mathrm{d}^{4} x \frac{a^{2}}{2}\left(\frac{\sqrt{3} \phi_{0}^{\prime} s_{0}}{\phi_{0}^{2}}\right)^{2}\left\{\psi^{\prime 2}-\left(2 \sqrt{2} \frac{\phi_{0}^{\prime}}{\phi_{0}}\right)^{2} \psi^{2}\right\},
$$

contains neither ghosts nor tachyons. These results indicate that perturbations in this theory are stable, and therefore its cosmological solutions are viable. Furthermore, we have explicitly solved for the evolution of the matter perturbation in this regime and found it to be completely suppressed when the dilaton vanishes (i.e. when gravity is maximally repulsive), hinting at a solution to the homogeneity problem.

\section{B. Future Work}

Here we have presented the most basic possible analysis of perturbations in cosmological solutions of this theory. However, there is a wealth of more detailed aspects of these solutions which we have neglected for the sake of simplicity, and which offer many potentially fruitful avenues of future investigation.

First, there is the fact that we have only been working with the action (2) with a vanishing potential $V(\phi, s)$, and without any radiative matter. Both the potential and radiative matter are important for the background cosmology which the authors of [4 12] have in mind and hence further insight may be gained by repeating the procedure in this paper with $V$ kept as an arbitrary function and/or with simple choices of interactions; a conformally-extended symmetry-breaking-type potential of the form

$$
V=\frac{\lambda}{4}\left(\frac{s^{2}}{2}-\alpha^{2} \phi^{2}\right)^{2}+\frac{\lambda^{\prime}}{4} \phi^{4}
$$

(motivated from [10]) would be one of the first obvious options to try — with $\lambda, \lambda^{\prime}$, and $\alpha$ here denoting coupling constants.

Second, we have not considered anisotropies in our analysis. Yet, as mentioned in the introduction, the inclusion of anisotropies is what leads to a (potential-independent) attractor mechanism which forces cosmological solutions of this theory through the origin of field space (with a loop in the $\mathbb{G}_{-}$region between Crunch and Bang, illustrated by the red curve in Figure 1). Moreover, the total suppression of perturbations (portrayed in Figure 22 about mid-way through the (repulsive gravity) period between the (attractive gravity) contraction 
and expansion epochs of the Universe in this theory appears to be novel; in other "matter bounce" scenarios this is not the case - but, usually because of anisotropies. These two reasons together offer strong motivation for expanding the present analysis to include anisotropies, and thereby determine precisely to what extent they play a role at the perturbative level.

Third, we have only considered the evolution of scalar metric perturbations. In cosmologies based on Einstein gravity, it is the scalar metric sector in which the strongest instabilities tend to occur. However, in the background theory we are considering the gravitational wave sector may also show instabilities which need to be analyzed. Thus, it will be important to study the behaviour of the other two classes of metric perturbations, namely vector and tensor perturbations, before drawing definite conclusions about the stability of the model. The study of tensor perturbations is of particular interest since it will yield the predictions of the model for the spectrum of gravitational waves.

Fourth, our treatment has been a purely classical one. This leaves the door open for a quantum analysis of this problem.

\section{Acknowledgements}

This work was supported by the Natural Sciences and Engineering Research Council of Canada. We would like to thank E. Ferreira and H. Bazrafshan Moghaddam for helpful discussions. We are particularly grateful to Paul Steinhardt for his careful reading of the manuscript and for his insightful comments.

\section{Appendix A: Consistency Check}

We can eliminate one of the two scalar field degrees of freedom via the following reparametrizations of the dilaton and the Higgs, respectively:

$$
\left\{\begin{array}{l}
\phi_{\mathrm{E}}=\sqrt{6} \cosh (\sigma / \sqrt{6}), \\
s_{\mathrm{E}}=\sqrt{6} \sinh (\sigma / \sqrt{6}),
\end{array}\right.
$$

in terms of a single scalar field $\sigma$. Setting $\phi=\phi_{\mathrm{E}}$ and $s=s_{\mathrm{E}}$, the Weyl-symmetric theory (2) reduces to ordinary Einstein gravity minimally coupled to this single scalar field,

$$
S_{\mathrm{E}}\left[\sigma ; g_{\mu \nu}\right]=\int \mathrm{d}^{4} x \sqrt{-g}\left\{-\frac{1}{2} R+\frac{1}{2} g^{\mu \nu} \nabla_{\mu} \sigma \nabla_{\nu} \sigma\right\}
$$

Moreover, setting $\phi=\phi_{\mathrm{E}}$ and $s=s_{\mathrm{E}}$ in the gravitational (3) and matter (5) equations of motion reduces them, as expected, to the usual Einstein equation and the massless Klein-Gordon equation for the scalar field $\sigma$, respectively:

$$
\begin{gathered}
G_{\alpha \beta}=-T_{\alpha \beta}, \\
g^{\mu \nu} \nabla_{\mu} \nabla_{\nu} \sigma=0 .
\end{gathered}
$$

Now, in order to recover the results of standard cosmological perturbation theory in Einstein gravity - where the perturbed metric (6) is the same - we need to choose appropriate reparametrizations of $\phi_{0}, \Pi, s_{0}$, and $\Theta$ such that, in this limit, the full dilaton and Higgs solutions become $\phi=\phi_{\mathrm{E}}$ and $s=s_{\mathrm{E}}$. Moreover, the scalar field $\sigma$ in terms of which $\phi_{\mathrm{E}}$ and $s_{\mathrm{E}}$ are written A1 should itself decompose, as usual, into a homogeneous (purely time-dependent) part $\sigma_{0}(\tau)$ plus a (spacetime-dependent) perturbation $\Delta(\tau, \mathbf{x})$, i.e.

$$
\sigma=\sigma_{0}+\varepsilon \Delta \text {. }
$$

The way to achieve this is to Taylor expand $\phi_{\mathrm{E}}=\sqrt{6} \cosh (\sigma / \sqrt{6})$ and $s_{\mathrm{E}}=\sqrt{6} \sinh (\sigma / \sqrt{6})$ in $\sigma$ about the background value $\sigma_{0}$, and match the resulting series, in powers of $\varepsilon$, with $\phi=\phi_{0}+\varepsilon \Pi$ and $s=s_{0}+\varepsilon \Theta$, respectively. This entails that the appropriate reparametrizations that recover the Einstein limit for the background fields are simply

$$
\left\{\begin{array}{l}
\phi_{0 \mathrm{E}}=\sqrt{6} \cosh \left(\sigma_{0} / \sqrt{6}\right) \\
s_{0 \mathrm{E}}=\sqrt{6} \sinh \left(\sigma_{0} / \sqrt{6}\right)
\end{array}\right.
$$


while for their respective perturbations, they must be

$$
\begin{aligned}
\Pi_{\mathrm{E}} & =\sum_{n=0}^{\infty} \frac{\sinh \left(\sigma_{0} / \sqrt{6}\right)}{6^{n}(2 n+1) !}\left[1+\frac{\operatorname{coth}\left(\sigma_{0} / \sqrt{6}\right)}{\sqrt{6}(2 n+2)} \varepsilon \Delta\right] \varepsilon^{2 n} \Delta^{2 n+1} \\
& =\sinh \left(\frac{\sigma_{0}}{\sqrt{6}}\right) \Delta+\frac{1}{2 \sqrt{6}} \cosh \left(\frac{\sigma_{0}}{\sqrt{6}}\right) \varepsilon \Delta^{2}+\mathcal{O}\left(\varepsilon^{2}\right)
\end{aligned}
$$

and

$$
\begin{aligned}
\Theta_{\mathrm{E}} & =\sum_{n=0}^{\infty} \frac{\cosh \left(\sigma_{0} / \sqrt{6}\right)}{6^{n}(2 n+1) !}\left[1+\frac{\tanh \left(\sigma_{0} / \sqrt{6}\right)}{\sqrt{6}(2 n+2)} \varepsilon \Delta\right] \varepsilon^{2 n} \Delta^{2 n+1} \\
& =\cosh \left(\frac{\sigma_{0}}{\sqrt{6}}\right) \Delta+\frac{1}{2 \sqrt{6}} \sinh \left(\frac{\sigma_{0}}{\sqrt{6}}\right) \varepsilon \Delta^{2}+\mathcal{O}\left(\varepsilon^{2}\right) .
\end{aligned}
$$

Upon substituting $\phi_{0}=\phi_{0 \mathrm{E}}, \Pi=\Pi_{\mathrm{E}}, s_{0}=s_{0 \mathrm{E}}$ and $\Theta=\Theta_{\mathrm{E}}$, which turn (8) into

$$
\left\{\begin{array}{l}
z_{\mathrm{E}}=6 \\
\tilde{z}_{\mathrm{E}}=-\sigma_{0}^{\prime 2}
\end{array}\right.
$$

and (9) into

$$
\left\{\begin{array} { l } 
{ \alpha _ { \mathrm { E } } = \varepsilon \frac { 1 } { 2 } \Delta ^ { 2 } + \ldots } \\
{ \tilde { \alpha } _ { \mathrm { E } } = - \sigma _ { 0 } ^ { \prime } \Delta ^ { \prime } - \varepsilon \frac { 1 } { 1 2 } \sigma _ { 0 } ^ { \prime 2 } \Delta ^ { 2 } + \ldots , }
\end{array} \quad \text { and } \quad \left\{\begin{array}{l}
\Upsilon_{\mathrm{E}}=6 \Phi-\varepsilon \frac{1}{2} \Delta^{2}+\ldots \\
\tilde{\Upsilon}_{\mathrm{E}}=-\sigma_{0}^{\prime 2} \Phi+\sigma_{0}^{\prime} \Delta^{\prime}+\varepsilon \frac{1}{12} \sigma_{0}^{\prime 2} \Delta^{2}+\ldots
\end{array}\right.\right.
$$

where $\cdots$ denotes higher order terms in $\varepsilon$, all of the equations of motion given in Table $\mathbb{I}$ reduce to their appropriate counterparts in the usual Einstein case [15], given in Table III]

\begin{tabular}{|l||l|l|}
\hline EOM & $\mathcal{O}(1)$ & $\mathcal{O}(\varepsilon)$ \\
\hline A3 time-time & $0=\mathcal{H}^{2}-\frac{1}{6} \sigma_{0}^{\prime 2}$ & $0=\nabla^{2} \Phi-3 \mathcal{H}\left(\Phi^{\prime}+\mathcal{H} \Phi\right)+\frac{1}{2} \sigma_{0}^{\prime 2} \Phi-\frac{1}{2} \sigma_{0}^{\prime} \Delta^{\prime}$ \\
\hline A3 space-space & $0=2 \mathcal{H}^{\prime}+\mathcal{H}^{2}+\frac{1}{2} \sigma_{0}^{\prime 2}$ & $0=\Phi^{\prime \prime}+3 \mathcal{H} \Phi^{\prime}+\left(2 \mathcal{H}^{\prime}+\mathcal{H}^{2}\right) \Phi+\frac{1}{2} \sigma_{0}^{\prime 2} \Phi-\frac{1}{2} \sigma_{0}^{\prime} \Delta^{\prime}$ \\
\hline A3 time-space & Trivial. & $0=\partial_{i}\left(\mathcal{H} \Phi+\Phi^{\prime}-\frac{1}{2} \sigma_{0}^{\prime} \Delta\right)$ \\
\hline A4 & $0=\sigma_{0}^{\prime \prime}+2 \mathcal{H} \sigma_{0}^{\prime}$ & $0=\Delta^{\prime \prime}+2 \mathcal{H} \Delta^{\prime}-\nabla^{2} \Delta-4 \sigma_{0}^{\prime} \Phi^{\prime}$ \\
\hline
\end{tabular}

Table III: Perturbed equations of motion in Einstein gravity.

Moreover, the second order action (11) reduces correctly [15] in the Einstein limit:

$$
S_{\mathrm{E}}^{(2)}=\varepsilon^{2} \int \mathrm{d}^{4} x \frac{a^{2}}{2}\left\{-6\left[\Phi^{\prime 2}+\frac{1}{3}(\nabla \Phi)^{2}\right]+\Delta^{\prime 2}-(\nabla \Delta)^{2}+8 \sigma_{0}^{\prime} \Phi^{\prime} \Delta\right\} .
$$

Because of the $\mathcal{O}(\varepsilon)$ terms appearing in $\Pi_{\mathrm{E}}$ and $\Theta_{\mathrm{E}}$, one may worry about possible $\mathcal{O}\left(\varepsilon^{2}\right)$ contributions to this limit from the first order action, which can be simplified to:

$$
S^{(1)}=\varepsilon \int \mathrm{d}^{4} x \frac{a^{2}}{2}\left\{-z \Phi^{\prime \prime}+2\left[\left(\mathcal{H}^{2}+\mathcal{H}^{\prime}\right) \alpha-\tilde{\alpha}\right]\right\} .
$$

Indeed, the $\alpha$ and $\tilde{\alpha}$ terms do contain $\mathcal{O}(\varepsilon)$ contributions in the Einstein limit as per (A12), leading a priori to $\mathcal{O}\left(\varepsilon^{2}\right)$ additions to A13 from A14. However, it is possible to verify that the entire term appearing in the square brackets in A14) actually vanishes at $\mathcal{O}(\varepsilon)$ in the Einstein limit by virtue of the background equations.

\section{Appendix B: Perturbed Action Coefficients}

For convenience, we define:

$$
\stackrel{*}{u}=\bar{u}^{\prime}-v \bar{u},
$$


as well as:

$$
\begin{aligned}
\bar{A} & =\tilde{A}^{\prime}+A \tilde{B} \\
\bar{B} & =\tilde{B}^{\prime}+B \tilde{B} \\
\bar{C} & =\tilde{C}^{\prime}+C \tilde{D} \\
\bar{D} & =\tilde{D}^{\prime}+D \tilde{D} \\
D^{*} & =-6 \mathcal{H} \tilde{u}^{\prime} D-3\left(\tilde{u}^{\prime} D\right)^{\prime} \\
\mathfrak{D}_{1} & =2 \bar{u} \hat{v}-6 \bar{u} \tilde{u}^{\prime} D-2 \phi_{0} u^{\prime} \\
\mathfrak{D}_{2} & =3 \tilde{u}^{\prime} D-\hat{v}-9 u^{\prime 2} \\
E & =\tilde{u}\left[\bar{A}^{2}-2 \tilde{A}(\mathcal{H} \bar{A}+A \bar{B})\right]-(\tilde{u} \tilde{A} \bar{A})^{\prime} \\
F & =\tilde{u}\left[\bar{C}^{2}-2 \tilde{C}(\mathcal{H} \bar{C}+C \bar{D})\right]-(\tilde{u} \tilde{C} \bar{C})^{\prime} \\
G & =2 \tilde{u}[(\bar{A} \bar{C}-C \tilde{A} \bar{D})-\tilde{C}(2 \mathcal{H} \bar{A}+A \bar{B})]-2(\tilde{u} \bar{A} \tilde{C})^{\prime}, \\
I & =2 \tilde{u} \bar{B}[\bar{A}-(2 \mathcal{H}+B) \tilde{A}]-2(\tilde{u} \tilde{A} \bar{B})^{\prime} \\
J & =2 \tilde{u} \bar{D}[\bar{A}-(2 \mathcal{H}+D) \tilde{A}]-2(\tilde{u} \tilde{A} \bar{D})^{\prime} \\
K & =2 \tilde{u} \bar{B}[\bar{C}-(2 \mathcal{H}+B) \tilde{C}]-2(\tilde{u} \tilde{C} \bar{B})^{\prime} \\
L & =2 \tilde{u} \bar{D}[\bar{C}-(2 \mathcal{H}+D) \tilde{C}]-2(\tilde{u} \tilde{C} \bar{D})^{\prime}
\end{aligned}
$$

Inserting $\Theta$ as given by 21 into 18 we get, after simplifications:

$$
\begin{aligned}
& S^{(2)}=\varepsilon^{2} \int \mathrm{d}^{4} x \frac{a^{2}}{2}\left\{\tilde{u} \tilde{A}^{2} \tilde{\Phi}^{\prime 2}+\tilde{u} \tilde{C}^{2} \tilde{\psi}^{\prime 2}+2 \tilde{u} \tilde{A} \tilde{C} \tilde{\Phi}^{\prime} \tilde{\psi}^{\prime}+p \tilde{\Phi}^{\prime} \tilde{\psi}+2 q \tilde{\Phi} \tilde{\psi}+m_{1} \tilde{\Phi}^{2}+m_{2} \tilde{\psi}^{2}\right. \\
& \left.+\kappa_{1} \Phi^{\prime} \psi+\kappa_{2} \Phi^{2}+\kappa_{3} \psi^{2}+\kappa_{4} \Phi \psi\right\}
\end{aligned}
$$

where

$$
\begin{aligned}
p & =2 \tilde{u}(\tilde{A} \bar{C}-\bar{A} \tilde{C})+3 \tilde{u}^{\prime} C \tilde{A} \\
2 q & =G+2 \hat{u} \tilde{A} \tilde{C}+3 \tilde{u}^{\prime} C \bar{A}+A C \mathfrak{D}_{1}, \\
m_{1} & =E+\hat{u} \tilde{A}^{2}+\frac{5}{3} A^{2} z \\
m_{2} & =F+\hat{u} \tilde{C}^{2}+3 \tilde{u}^{\prime} C(\bar{C}+\mathcal{H} C)+\frac{3}{2}\left(\tilde{u}^{\prime} C^{2}\right)^{\prime}+C^{2} \mathfrak{D}_{2},
\end{aligned}
$$

and

$$
\begin{aligned}
& \kappa_{1}=\frac{J}{A}-\frac{K}{C}+2 \hat{u}(\tilde{B}+2 \bar{u} \tilde{D})-6 \bar{u} \bar{v} \tilde{u}^{\prime}-\hat{v} \stackrel{*}{u}+2 \bar{u} \stackrel{*}{D}-3 \tilde{u}^{\prime}(\bar{B}-\stackrel{*}{u} D)+2 \phi_{0} v u^{\prime}+(D-B) \mathfrak{D}_{1}, \\
& \kappa_{2}=\tilde{u} \bar{B}^{2}-\frac{I}{A}(\mathcal{H}+B)-\frac{1}{2}\left(\frac{I}{A}\right)^{\prime}+\hat{u} \tilde{B}(\tilde{B}-4 \bar{u}[\mathcal{H}+B])-2(\hat{u} \bar{u} \tilde{B})^{\prime}-\frac{5}{3}\left((B+2 \mathcal{H}) B z+(B z)^{\prime}\right), \\
& \kappa_{3}=\tilde{u} \bar{D}^{2}-\frac{L}{C}(\mathcal{H}+D)-\frac{1}{2}\left(\frac{L}{C}\right)^{\prime}+\hat{u} \tilde{D}(\tilde{D}+2[\mathcal{H}+D])+(\hat{u} \tilde{D})^{\prime}-12 \tilde{v} \bar{v} \tilde{u}^{\prime}-\frac{3}{2}\left(\bar{v} \tilde{u}^{\prime}\right)^{\prime} \\
& -\mathcal{H} \hat{v}(3 \tilde{v}+v)-\frac{1}{2}(\hat{v}[3 \tilde{v}+v])^{\prime}+4 \tilde{v} \stackrel{*}{D}-3 \tilde{u}^{\prime}\left(D \bar{D}+\mathcal{H}\left[\tilde{D}^{\prime}-D^{2}\right]\right)-\frac{1}{2}\left(3 \tilde{u}^{\prime}\left[\tilde{D}^{\prime}-D^{2}\right]-\stackrel{D}{ }^{\prime}\right. \\
& +9 \bar{v} u^{\prime 2}-(D+2 \mathcal{H}) D \mathfrak{D}_{2}-\left(D \mathfrak{D}_{2}\right)^{\prime} \\
& \kappa_{4}=2 \tilde{u} \bar{B} \bar{D}-B \frac{J}{A}-\frac{K}{C}(2 \mathcal{H}+D)-\left(\frac{K}{C}\right)^{\prime}+2 \hat{u}([\mathcal{H}+4 \tilde{v}] \tilde{B}-2 \bar{u} B \tilde{D})+2(\hat{u} \tilde{B})^{\prime}-3 \bar{v} \tilde{u}^{\prime} u^{*} \\
& -2 \mathcal{H} \hat{v} \stackrel{*}{u}-(\hat{v} \stackrel{*}{u})^{\prime}+\stackrel{*}{u} \stackrel{*}{D}-3 \tilde{u}^{\prime}(D \bar{B}+2 \mathcal{H}[\bar{B}-\stackrel{*}{u} D])-3\left(\tilde{u}^{\prime}[\bar{B}-\stackrel{*}{u} D]\right)^{\prime}-24 \phi_{0} u^{\prime}\left(\tilde{v}^{\prime}+\tilde{v}^{2}\right) \\
& -(D+2 \mathcal{H}) B \mathfrak{D}_{1}-\left(B \mathfrak{D}_{1}\right)^{\prime} \text {. }
\end{aligned}
$$


Then, the four ODEs that the functions $A, B, C$, and $D$ need to satisfy in order for $S^{(2)}$ to be placed in the form 22 are:

$$
\begin{aligned}
& \kappa_{1}=0, \\
& \kappa_{2}=0, \\
& \kappa_{3}=0, \\
& \kappa_{4}=0 .
\end{aligned}
$$

\section{Appendix C: Perturbed Action Diagonalization}

We can write the second order action 222 as:

$$
\begin{aligned}
S^{(2)} & =\varepsilon^{2} \int \mathrm{d}^{4} x \frac{a^{2}}{2}\left\{\tilde{u}\left(\left[\begin{array}{ll}
\tilde{\Phi}^{\prime} & \tilde{\psi}^{\prime}
\end{array}\right]\left[\begin{array}{cc}
\tilde{A}^{2} & \tilde{A} \tilde{C} \\
\tilde{A} \tilde{C} & \tilde{C}^{2}
\end{array}\right]\left[\begin{array}{c}
\tilde{\Phi}^{\prime} \\
\tilde{\psi}^{\prime}
\end{array}\right]\right)+p \tilde{\Phi}^{\prime} \tilde{\psi}+2 q \tilde{\Phi} \tilde{\psi}+m_{1} \tilde{\Phi}^{2}+m_{2} \tilde{\psi}^{2}\right\} \\
& =\varepsilon^{2} \int \mathrm{d}^{4} x \frac{a^{2}}{2}\left\{\tilde{u}\left(\mathbf{f}^{\mathrm{T}}\right)^{\prime} \mathbf{K} \mathbf{f}^{\prime}+p \tilde{\Phi}^{\prime} \tilde{\psi}+2 q \tilde{\Phi} \tilde{\psi}+m_{1} \tilde{\Phi}^{2}+m_{2} \tilde{\psi}^{2}\right\}
\end{aligned}
$$

where we have defined

$$
\mathbf{f}=\left[\begin{array}{c}
\tilde{\Phi} \\
\tilde{\psi}
\end{array}\right], \quad \mathbf{K}=\left[\begin{array}{ll}
\tilde{A}^{2} & \tilde{A} \tilde{C} \\
\tilde{A} \tilde{C} & \tilde{C}^{2}
\end{array}\right]
$$

We can diagonalize the kinetic matrix as:

$$
\mathbf{K}=\left[\begin{array}{cc}
\tilde{A} & -\tilde{C} \\
\tilde{C} & \tilde{A}
\end{array}\right]\left[\begin{array}{ll}
1 & 0 \\
0 & 0
\end{array}\right]\left[\begin{array}{cc}
\tilde{A} & \tilde{C} \\
-\tilde{C} & \tilde{A}
\end{array}\right] .
$$

So, if we define

$$
\mathbf{Q}=\left[\begin{array}{cc}
\tilde{A} & \tilde{C} \\
-\tilde{C} & \tilde{A}
\end{array}\right]
$$

this suggests the field redefinition:

$$
\tilde{\mathbf{f}}=\left[\begin{array}{l}
\zeta \\
\xi
\end{array}\right]=\mathbf{Q f}=\left[\begin{array}{cc}
\tilde{A} & \tilde{C} \\
-\tilde{C} & \tilde{A}
\end{array}\right]\left[\begin{array}{c}
\tilde{\Phi} \\
\tilde{\psi}
\end{array}\right]=\left[\begin{array}{c}
\tilde{A} \tilde{\Phi}+\tilde{C} \tilde{\psi} \\
-\tilde{C} \tilde{\Phi}+\tilde{A} \tilde{\psi}
\end{array}\right]
$$

Inserting this into $S^{(2)}$ diagonalizes the kinetic term. After simplifying and integrating by parts, we can write it as:

$$
\begin{aligned}
S^{(2)} & =\varepsilon^{2} \int \mathrm{d}^{4} x \frac{a^{2}}{2}\left\{\tilde{u}\left(\tilde{\mathbf{f}}^{\prime}\right)^{\mathrm{T}}\left[\begin{array}{ll}
1 & 0 \\
0 & 0
\end{array}\right] \tilde{\mathbf{f}}^{\prime}+\tilde{\mathbf{f}}^{\mathrm{T}} \mathbf{M} \tilde{\mathbf{f}}+\left(\tilde{\mathbf{f}}^{\prime}\right)^{\mathrm{T}} \mathbf{N} \tilde{\mathbf{f}}\right\} \\
& =\varepsilon^{2} \int \mathrm{d}^{4} x \frac{a^{2}}{2}\left\{\tilde{u} \zeta^{\prime 2}+\tilde{\mathbf{f}}^{\mathrm{T}} \mathbf{M} \tilde{\mathbf{f}}+\left(\tilde{\mathbf{f}}^{\prime}\right)^{\mathrm{T}} \mathbf{N} \tilde{\mathbf{f}}\right\},
\end{aligned}
$$

where, if we define for convenience

$$
\mathcal{A}=\frac{1}{\left(\tilde{A}^{2}+\tilde{C}^{2}\right)},
$$

then

$$
\mathbf{N}=\left[\begin{array}{ll}
N_{1} & N_{2} \\
N_{3} & N_{4}
\end{array}\right],
$$

with

$$
\begin{aligned}
& N_{1}=\mathcal{A}^{2}\left[p \tilde{A} \tilde{C}-2 \tilde{u} \frac{1}{\mathcal{A}}\left(\tilde{A} \tilde{A}^{\prime}+\tilde{C} \tilde{C}^{\prime}\right)\right], \\
& N_{2}=\mathcal{A}^{2}\left[p \tilde{A}^{2}-2 \tilde{u} \frac{1}{\mathcal{A}}\left(\tilde{A} \tilde{C}^{\prime}-\tilde{C} \tilde{A}^{\prime}\right)\right], \\
& N_{3}=\mathcal{A}^{2}\left[-p \tilde{C}^{2}\right], \\
& N_{4}=\mathcal{A}^{2}[-p \tilde{A} \tilde{C}],
\end{aligned}
$$


and

$$
\mathbf{M}=\left[\begin{array}{ll}
M_{1} & M_{2} \\
M_{3} & M_{4}
\end{array}\right]
$$

with

$$
\begin{aligned}
& M_{1}=\mathcal{A}^{2}\left[\tilde{u}\left(\tilde{A} \tilde{A}^{\prime}+\tilde{C} \tilde{C}^{\prime}\right)^{2}+m_{1} \tilde{A}^{2}+m_{2} \tilde{C}^{2}+2 q \tilde{A} \tilde{C}+\frac{p}{\mathcal{A}} \tilde{C}(\mathcal{A} \tilde{A})^{\prime}\right], \\
& M_{2}=\mathcal{A}^{2}\left[\left(\tilde{A}^{2}-\tilde{C}^{2}\right)\left(\tilde{u} \tilde{A}^{\prime} \tilde{C}^{\prime}+q\right)+\tilde{A} \tilde{C}\left(\tilde{u}\left[\tilde{C}^{\prime 2}-\tilde{A}^{\prime 2}\right]+m_{2}-m_{1}\right)+\frac{p}{\mathcal{A}} \tilde{A}(\mathcal{A} \tilde{A})^{\prime}\right], \\
& M_{3}=\mathcal{A}^{2}\left[\left(\tilde{A}^{2}-\tilde{C}^{2}\right)\left(\tilde{u} \tilde{A}^{\prime} \tilde{C}^{\prime}+q\right)+\tilde{A} \tilde{C}\left(\tilde{u}\left[\tilde{C}^{\prime 2}-\tilde{A}^{\prime 2}\right]+m_{2}-m_{1}\right)-\frac{p}{\mathcal{A}} \tilde{C}(\mathcal{A} \tilde{C})^{\prime}\right], \\
& M_{4}=\mathcal{A}^{2}\left[\tilde{u}\left(\tilde{A} \tilde{C}^{\prime}-\tilde{C} \tilde{A}^{\prime}\right)^{2}+m_{1} \tilde{C}^{2}+m_{2} \tilde{A}^{2}-2 q \tilde{A} \tilde{C}-\frac{p}{\mathcal{A}} \tilde{A}(\mathcal{A} \tilde{C})^{\prime}\right] .
\end{aligned}
$$

So, if we further define

$$
\begin{aligned}
c_{1} & =M_{1}-\mathcal{H} N_{1}-\frac{1}{2} N_{1}^{\prime}, \\
c_{2} & =M_{4}-\mathcal{H} N_{4}-\frac{1}{2} N_{4}^{\prime}, \\
c_{3} & =M_{2}+M_{3}-2 \mathcal{H} N_{3}-N_{3}^{\prime}, \\
c_{4} & =N_{2}-N_{3},
\end{aligned}
$$

then (C8) yields precisely (24).

\section{Appendix D: Deep Antigravity Full Solution}

Upon substituting the background solution $(34)$ for $\phi_{0}$ from 8 , using the properties of Jacobi elliptic functions and dropping the $\mathcal{O}(1)$ terms in $\phi_{0}$, our full ODE $[33$ for the perturbation $\psi$ becomes:

$$
0=\frac{\mathrm{d}^{2}}{\mathrm{~d} \eta^{2}} \psi+\left[-4 \frac{\mathrm{cn}(\eta, m)}{\operatorname{sn}(\eta, m) \mathrm{dn}(\eta, m)}\right] \frac{\mathrm{d}}{\mathrm{d} \eta} \psi+\left[2 \sqrt{2} \frac{\mathrm{cn}(\eta, m)}{\operatorname{sn}(\eta, m) \mathrm{dn}(\eta, m)}\right]^{2} \psi .
$$

This has an exact solution:

$$
\begin{aligned}
& \psi=(1-\left.m^{2} \mathrm{sn}^{2}(\eta, m)\right)^{-\frac{13+\mathrm{i} \sqrt{23}}{2(1+\mathrm{i} \sqrt{23})}}\left(-\mathrm{sn}^{2}(\eta, m)\right)^{\frac{5}{4}+\mathrm{i} \frac{\sqrt{7}}{4}} \\
& \times \mathscr{C}_{1} \mathrm{H}\left(1-\frac{1}{m^{2}}, \frac{[2+\mathrm{i}(\sqrt{7}+\sqrt{23})]\left[4 m^{2}-2+\mathrm{i}(\sqrt{23}-\sqrt{7})\right]}{32 m^{2}},\right. \\
&\left.1+\mathrm{i} \frac{\sqrt{7}+\sqrt{23}}{4}, \frac{1}{2}+\mathrm{i} \frac{\sqrt{7}+\sqrt{23}}{4}, \frac{1}{2}, 1+\mathrm{i} \frac{\sqrt{7}}{2}, 1-\mathrm{sn}^{2}(\eta, m)\right) \\
&+\mathscr{C}_{2} \operatorname{cn}(\eta, m) \mathrm{H}\left(1-\frac{1}{m^{2}},[10+3 \mathrm{i}(\sqrt{7}+\sqrt{23})]\right. \\
& \quad \times \frac{\left[1324 m^{2}-477-9 \sqrt{7} \sqrt{23}+\mathrm{i}(162 \sqrt{23}-192 \mathrm{i} \sqrt{7})\right]}{10592 m^{2}}, \\
&\left.\left.\quad \frac{3}{2}+\mathrm{i} \frac{\sqrt{7}+\sqrt{23}}{4}, 1+\mathrm{i} \frac{\sqrt{7}+\sqrt{23}}{4}, \frac{3}{2}, 1+\mathrm{i} \frac{\sqrt{7}}{2}, 1-\mathrm{sn}^{2}(\eta, m)\right)\right\},
\end{aligned}
$$

where $\mathrm{H}$ is the Heun general function, and $\mathscr{C}_{1}$ and $\mathscr{C}_{2}$ are constants.

[1] S. Hawking and R. Penrose, Proc.Roy.Soc.Lond. A314, 529 (1970).

[2] S. Hawking and G. Ellis, The Large Scale Structure of Space-Time (Cambridge University Press, Cambridge, 1973).

[3] R. M. Wald, General Relativity (University Of Chicago Press, Chicago, 1973).

[4] I. Bars and S.-H. Chen, Phys.Rev. D83, 043522 (2011), 1004.0752. 
[5] I. Bars, S.-H. Chen, and N. Turok, Phys.Rev. D84, 083513 (2011), 1105.3606.

[6] I. Bars (2011), 1109.5872.

[7] I. Bars, S.-H. Chen, P. J. Steinhardt, and N. Turok, Phys.Lett. B715, 278 (2012), 1112.2470.

[8] I. Bars, S.-H. Chen, P. J. Steinhardt, and N. Turok, Phys.Rev. D86, 083542 (2012), 1207.1940.

[9] I. Bars (2012), 1209.1068.

[10] I. Bars, P. J. Steinhardt, and N. Turok, Phys.Rev. D89, 043515 (2014), 1307.1848.

[11] I. Bars, P. J. Steinhardt, and N. Turok, Phys.Lett. B726, 50 (2013), 1307.8106.

[12] I. Bars, P. J. Steinhardt, and N. Turok (2013), 1312.0739.

[13] J. M. Cline, S. Jeon, and G. D. Moore, Phys. Rev. D 70, 043543 (2004).

[14] P. Caputa, S. S. Haque, J. Olson, and B. Underwood, Class.Quant.Grav. 30, 195013 (2013), 1306.0012.

[15] V. Mukhanov, H. Feldman, and R. Brandenberger, Phys. Rept. 215, 203 (1992).

[16] R. H. Brandenberger, Lect.NotesPhys. 646, 127 (2004).

[17] A. Padilla, D. Stefanyszyn, and M. Tsoukalas, Phys.Rev. D89, 065009 (2014), 1312.0975.

[18] A. D. Linde, Pisma Zh.Eksp.Teor.Fiz. 30, 479 (1979).

[19] A. Starobinsky, Pisma Astron. Zh. 7, 67 (1981).

[20] T. Futamase, T. Rothman, and R. Matzner, Phys.Rev. D39, 405 (1989).

[21] L. R. Abramo, L. Brenig, E. Gunzig, and A. Saa, Phys.Rev. D67, 027301 (2003), gr-qc/0210069.

[22] J. J. M. Carrasco, W. Chemissany, and R. Kallosh, JHEP 1401, 130 (2014), 1311.3671.

[23] I. Bars, P. Steinhardt, and N. Turok, Phys.Rev. D89, 061302 (2014), 1312.0739.

[24] See also [17] for a discussion of more general two field scale-invariant theories coupled to gravity.

[25] In the context of single scalar field matter, models with non-minimal coupling between matter and gravity which allow for solutions to transit between the gravity and antigravity phase were studied in [18, but they were shown to have singularities and instabilities [19.21].

[26] Note that some curvature invariants blow up at the transition point between the gravity and antigravity phase (see e.g. 22] ). However, it was argued in [23] that this does not effect the viability of the geodesically complete solutions. 